\title{
Surface Josephson Plasma Waves in Layered Superconductors
}

\author{
Sergey Savel'ev, ${ }^{1}$ Valery Yampol'skii, ${ }^{1,2}$ and Franco Nori ${ }^{1,3}$ \\ ${ }^{1}$ Frontier Research System, The Institute of Physical and Chemical Research (RIKEN), Wako-shi, Saitama, 351-0198, Japan \\ ${ }^{2}$ A. Ya. Usikov Institute for Radiophysics and Electronics, NASU, 61085 Kharkov, Ukraine \\ ${ }^{3}$ Physics Department, MCTP, CSCS, University of Michigan, Ann Arbor, Michigan 48109-1040, USA
}

(Received 10 August 2005; published 26 October 2005)

\begin{abstract}
We predict the existence of surface waves in layered superconductors in the $\mathrm{THz}$ frequency range, below the Josephson plasma frequency $\omega_{J}$. This wave propagates along the vacuum-superconductor interface and dampens in both transverse directions out of the surface (i.e., towards the superconductor and towards the vacuum). This is the first prediction of propagating surface waves in any superconductor. These predicted surface Josephson plasma waves are important for different phenomena, including the complete suppression of the specular reflection from a sample (Wood's anomalies) and a huge enhancement of the wave absorption (which can be used as a THz detector).
\end{abstract}

DOI: 10.1103/PhysRevLett.95.187002

PACS numbers: 74.78.Fk, 74.50.+r, 74.72.Hs

High temperature $\mathrm{Bi}_{2} \mathrm{Sr}_{2} \mathrm{CaCu}_{2} \mathrm{O}_{8+\delta} \quad(\mathrm{Bi} 2212)$ and $\mathrm{Tl}_{2} \mathrm{Ba}_{2} \mathrm{CaCu}_{2} \mathrm{O}_{8+\delta}$ ( $\mathrm{Tl2212}$ ) superconductors have a layered structure of superconducting $\mathrm{CuO}_{2}$ planes with Josephson coupling between them [1]. This structure favors the propagation of electromagnetic waves, called Josephson plasma waves (JPW) [2-4], through the layers. These waves attract considerable interest because of their Terahertz frequency range [from $300 \mathrm{GHz}$ to $30 \mathrm{THz}$ corresponding to $1000-90 \mu \mathrm{m}$ (wavelength)], which is still hardly reachable for both electronic and optical devices [5]. During the last decade there have been many attempts to push $\mathrm{THz}$ science and technology forward [5] because of many important applications in physics, astronomy, chemistry, biology, and medicine, including $\mathrm{THz}$ imaging, spectroscopy, tomography, medical diagnosis, health monitoring, environmental control, as well as chemical and biological identification.

The unusual optical properties of layered superconductors, including reflectivity and transmissivity, caused by the JPW excitation were studied in, e.g., Refs. [2,6-9]. In particular, Ref. [8] demonstrated that the spectrum of JPW consists of two branches due to a peculiar effect [10] of dynamical breaking of charge neutrality and, therefore, the transmissivity exhibits a sharp peak at frequencies just above the Josephson plasma frequency $\omega_{J}$.

All previous works on this problem have focused on running waves in the frequency range above the gap of the JPW spectrum, i.e., above the Josephson plasma frequency $\omega>\omega_{J}$. A similar gapped spectrum also appears in solid state plasma [11]. In such situations the presence of the sample boundary can produce a new branch of the wave spectrum inside the gap, i.e., a surface plasmon $[11,12]$. In general, surface waves play a very important role in many fundamental resonant phenomena, such as the "Wood anomalies" in the reflectivity $[12,13]$ and transmissivity [14] of periodically corrugated metal and semiconducting samples, and are employed in many devices. Therefore, it is important to know if surface waves can exist in layered superconductors.

Here, we prove that surface Josephson plasma waves can propagate along the surface between the superconductor and the vacuum in a wide frequency range below $\omega_{J}$. We derive the dispersion relation for these waves and propose ways to excite these. We show that these surface waves play an important role in the absorption and reflection of electromagnetic waves, including their resonance dependence on the incident angle $\theta$. The studied resonant absorbability could be experimentally observed by measurement of the surface impedance of a sample or dc resistivity. The predicted phenomena are potentially useful for designing $\mathrm{THz}$ detectors.

Surface Josephson plasma waves. -We consider a semiinfinite layered superconductor in the geometry shown in the inset in Fig. 1. Using a standard approximation, the spatial variations inside the very thin superconducting layers are neglected in the direction perpendicular to the

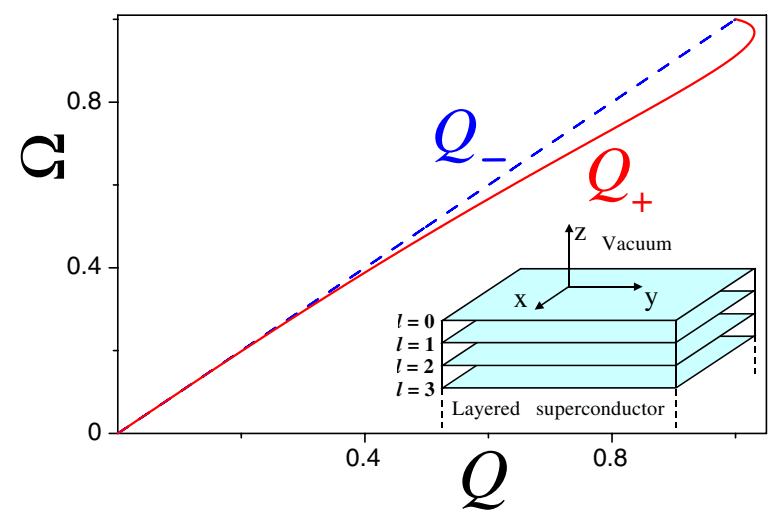

FIG. 1 (color online). Spectra of two branches " \pm " of the surface Josephson plasma waves for the parameters $\alpha=0.1$ and $\beta=1.4$, standard for $\mathrm{Bi} 2212$. The inset shows the geometry used. 
layers. The crystallographic ab plane coincides with the $\mathbf{x y}$ plane and the $\mathbf{c}$ axis is along the $z$ axis. Superconducting layers are numbered by the integer $l \geq 0$.

We consider a surface $p$ wave with the electric, $\vec{E}=$ $\left\{E_{x}, 0, E_{z}\right\}$, and magnetic, $\vec{H}=\{0, H, 0\}$, fields damped away from the interface $z=0$,

$$
H, E_{x}, E_{z} \propto \exp (-i \omega t+i q x-k l D)
$$

inside a layered superconductor, $z<0$, and

$$
H^{\mathrm{vac}}, E_{x}^{\mathrm{vac}}, E_{z}^{\mathrm{vac}} \propto \exp \left(-i \omega t+i q x-k_{\mathrm{vac}} z\right),
$$

in vacuum, for $z>0$ and $q>\omega / c$. Here $D$ is the spatial period of the layered structure.

The Maxwell equations for waves (2), $q H^{\mathrm{vac}}=$ $-(\omega / c) E_{z}^{\mathrm{vac}}, \quad k_{\mathrm{vac}} H^{\mathrm{vac}}=-(i \omega / c) E_{x}^{\mathrm{vac}}, \quad i q E_{z}^{\mathrm{vac}}+$ $k_{\mathrm{vac}} E_{x}^{\mathrm{vac}}=-(i \omega / c) H^{\mathrm{vac}}$, provide both the usual dispersion relation, $k_{\mathrm{vac}}=\sqrt{q^{2}-\omega^{2} / c^{2}}$, for the wave in vacuum and the ratio of amplitudes for tangential electric and magnetic fields at the interface $z=+0$ (i.e., right above the sample surface)

$$
\frac{E_{x}^{\mathrm{vac}}}{H^{\mathrm{vac}}}=\frac{i c}{\omega} \sqrt{q^{2}-\omega^{2} / c^{2}}
$$

Inside the layered superconductor, where $z<0$, the gauge-invariant phase difference is described by a set of coupled sine-Gordon equations. For Josephson plasma waves, these nonlinear equations can be linearized and rewritten in terms of the magnetic fields $H^{l}$ between the $l$ th and $(l+1)$ th layers,

$$
\begin{array}{r}
\left(1-\frac{\lambda_{a b}^{2}}{D^{2}} \partial_{l}^{2}\right)\left(\frac{\partial^{2} H^{l}}{\partial t^{2}}+\omega_{r} \frac{\partial H^{l}}{\partial t}+\omega_{J}^{2} H^{l}-\alpha \omega_{J}^{2} \partial_{l}^{2} H^{l}\right) \\
-\frac{c^{2}}{\varepsilon} \frac{\partial^{2} H^{l}}{\partial x^{2}}=0 .
\end{array}
$$

Here $\lambda_{a b}$ is the London penetration depth in the $z$ direction, the operator $\partial_{l}^{2}$ is defined as $\partial_{l}^{2} f_{l}=f_{l+1}+f_{l-1}-2 f_{l}$, $\omega_{J}=\sqrt{8 \pi e D J_{c} / \hbar \varepsilon}$ is the Josephson plasma frequency determined by the maximum Josephson current $J_{c}$ and dielectric constant $\varepsilon$, and $\omega_{r}$ is the relaxation frequency. The effect of breaking charge neutrality [10], which is crucial for our analysis, is taken into account in Eq. (4). The constant $\alpha$ characterizing this effect was estimated, e.g., in Ref. [8], $\alpha \sim 0.05-0.1$ for $\mathrm{Bi} 2212$ or Tl2212 crystals.

Substituting the wave (1) in Eq. (4), we obtain the implicit equation for the damped-wave transverse wave vector $k(q, \omega)$

$$
\begin{aligned}
\frac{\omega^{2}}{\omega_{J}^{2}}= & 1+\frac{\lambda_{c}^{2} q^{2}}{1-\left(4 \lambda_{a b}^{2} / D^{2}\right) \sinh ^{2}[k(q, \omega) D / 2]} \\
& -4 \alpha \sinh ^{2}[k(q, \omega) D / 2], \\
\lambda_{c}^{2}= & \frac{c^{2}}{\omega_{J}^{2} \varepsilon}
\end{aligned}
$$

for $\omega_{r} \rightarrow 0$. A similar spectrum, but for running Josephson plasma waves [with imaginary $k(q, \omega)$ ], was earlier obtained in Ref. [8]. At $\omega<\omega_{J}$, solving Eq. (5) with respect to $\sinh ^{2}[k(q, \omega) D / 2]$ results in two branches of positive transverse spatial decrement, $k_{ \pm}(q, \omega)>0$. It is important to stress that Eq. (5) is not a spectrum of the studied longitudinal surface waves. Indeed, Eq. (5) has two free parameters, $\omega$ and $q$. We need to obtain the $q(\omega)$ dispersion relation for our surface waves. This dispersion relation $q(\omega)$ can be obtained by joining fields (1) in a superconductor and (2) in vacuum at the sample surface via the boundary conditions. Thus, in order to find the spectrum of the surface Josephson plasma waves, we can derive the ratio $E_{x} / H$ in the superconductor at the sample surface and equate this ratio to Eq. (3).

The difference between the magnetic field $H^{\mathrm{vac}}$ in vacuum and the value $H^{0}$ between the 0 th and the 1 st superconducting layers is described by the London equation,

$$
\frac{H^{\mathrm{vac}}-H^{0}}{D} \approx \frac{A_{x 0}}{\lambda_{a b}^{2}} \approx \frac{-i c}{\lambda_{a b}^{2} \omega} E_{x 0} .
$$

Here $A_{x 0}$ and $E_{x 0}$ are the $x$ components of the vector potential and electric field in the first superconducting layer. We ignore the displacement current in Eq. (6) because it is proportional to a small parameter $\left(\lambda_{a b} \omega / c\right)^{2}$. Besides, we neglected the contribution of the gaugeinvariant scalar potential into the $x$ component of the electric field since the Debye length is much shorter than the wavelength $q^{-1}$. In order to eliminate $H^{0}$, we use the relation $H^{\mathrm{vac}}-H^{0}=H^{\mathrm{vac}}[1-\exp (-k D)]$, that follows from Eq. (1) with $l=0$ and $l=1$. Now, using Eq. (6), we obtain the ratio between electric and magnetic fields at $z=-0$ (i.e., right below the sample surface)

$$
\frac{E_{x}}{H^{\mathrm{vac}}}=\frac{i \omega \lambda_{a b}^{2}}{c D}[1-\exp (-k D)] .
$$

Using the continuity conditions for the tangential components of electric field at the surface and Eqs. (3), (5), and (7), we obtain the dispersion relation for two branches of the surface wave corresponding to two solutions of Eq. (5). For $\left(1-\omega / \omega_{J}\right) \gg \sqrt{\alpha / \varepsilon} D / \lambda_{a b} \approx 5 \times 10^{-4}$, this spectrum can be written as

$$
\begin{aligned}
& Q_{-}(\Omega)=\Omega \\
& Q_{+}(\Omega)=\Omega\left(1+\beta^{2} \Omega^{2} \Gamma_{\Omega}\left\{1+2 \Gamma_{\Omega}\left[1-\left(1+\Gamma_{\Omega}^{-1}\right)^{1 / 2}\right]\right\}\right)^{1 / 2}
\end{aligned}
$$

for two branches " $\mp$." Here we introduce the dimensionless variables: $Q=c q / \omega_{J}, \Omega=\omega / \omega_{J}, \beta=2 \lambda_{a b}^{2} \omega_{J} / c D$, and $\Gamma_{\Omega}=\left(1-\Omega^{2}\right) / 4 \alpha$. The value of the parameter $\beta$ for Bi2212 is about 1.4. The spectra Eq. (8) are shown in Fig. 1. Both branches merge in a narrow frequency region below $\omega_{J}$, i.e., $(1-\Omega) \sim(\alpha / \varepsilon)^{1 / 2}\left(D / \lambda_{a b}\right)$.

The surface mode "-" attenuates into the vacuum at very large distances, of about $c \lambda_{a b} / \omega D$, and seems to be 
difficult to observe experimentally. Another mode, "+", dampens on scales $\sim c / \omega$ and is of significant interest. As we show below, it plays an important role in the transmissivity and reflectivity properties of layered superconductors at frequencies $\omega<\omega_{J}$.

Excitation of the surface waves and resonant electromagnetic absorption. - One of the ways to excite surface waves is via externally applied electromagnetic waves on a sample having spatially modulated parameters. Thus, we consider a weak modulation of the maximum current density $J_{c}$ due to, say, creation of pancake vortices by the outof-plane magnetic field. This can result in the modulation of $\omega_{J}$. For simplicity, we assume that

$$
\omega_{J}^{2}(x)=\omega_{J}^{2}\left[1+2 \mu \cos \left(\frac{2 \pi x}{a}\right)\right], \quad \mu \ll 1,
$$

where $a$ is a spatial period.

An electromagnetic wave with $\omega<\omega_{J}$ incident at an angle $\theta$ with respect to the sample surface generates modes having longitudinal wave vectors $q_{m}=\omega \sin \theta / c+$ $2 \pi m / a$, with integer $m$. Almost all of these modes for $m \neq$ 0 are weak, because $\mu \ll 1$. However, one of these modes (e.g., for $m=1$ ) can be excited with large amplitude at resonance, i.e., when the wave vector $q_{1}=(\omega / c) \sin \theta+$ $2 \pi / a$ is close to the wave vector $q_{+}=\omega_{J} Q_{+}(\Omega) / c$ of the surface wave (8). This corresponds to the incident angle $\theta$ close to the resonance angle $\theta_{0}$ defined by

$$
\Omega \sin \theta_{0}+\frac{2 \pi c}{a \omega_{J}}=Q_{+}(\Omega) .
$$

Because of this resonance, the amplitude of the wave with $q=q_{1}$ can be of the same order, or even higher than, the amplitude $H_{\mathrm{in}}^{\mathrm{vac}}$ of the incident wave. In resonance, the mode with $q=q_{1}$ is actually the surface Josephson plasma wave discussed above.

Let us discuss the mechanism of excitation of surface waves. The incident $H_{\mathrm{in}}^{\mathrm{vac}}$ (red arrow in Fig. 2) and specularly-reflected $H_{\mathrm{sp}}^{\mathrm{vac}}$ (blue dashed arrow in Fig. 2) electromagnetic waves

$$
H_{\mathrm{in} ; \mathrm{sp}}^{\mathrm{vac}} \exp \left\{i\left(\frac{x \sin \theta \pm z \cos \theta}{c}-t\right) \omega\right\}
$$

generate the wave (in solid green, Fig. 2) damped inside the superconductor,

$$
H_{0} \exp \left\{i\left(\frac{x \sin \theta}{c}-t\right) \omega-k_{-}\left(\frac{\omega \sin \theta}{c}, \omega\right) D l\right\},
$$

having a lower $k_{-}(\Omega)$, compared to $k_{+}(\Omega)$ calculated from Eq. (5). Instead, as was shown in Ref. [8], the longer wave is mainly generated at $\left(\omega-\omega_{J}\right) / \omega_{J} \gg \sqrt{\alpha / \varepsilon} D / \lambda_{a b} \approx$ $5 \times 10^{-4}$. Moreover, at low frequencies, this longer wave exhibits a correct limiting behavior, producing magnetic fields described by the well-known London equation. The waves $H_{\mathrm{in}}^{\mathrm{vac}}, H_{\mathrm{sp}}^{\mathrm{vac}} \approx H_{\mathrm{in}}^{\mathrm{vac}}$, and $H_{0} \approx 2 H_{\mathrm{in}}^{\mathrm{vac}}$ represent the solution of the problem in the zero approximation with respect to $\mu$.
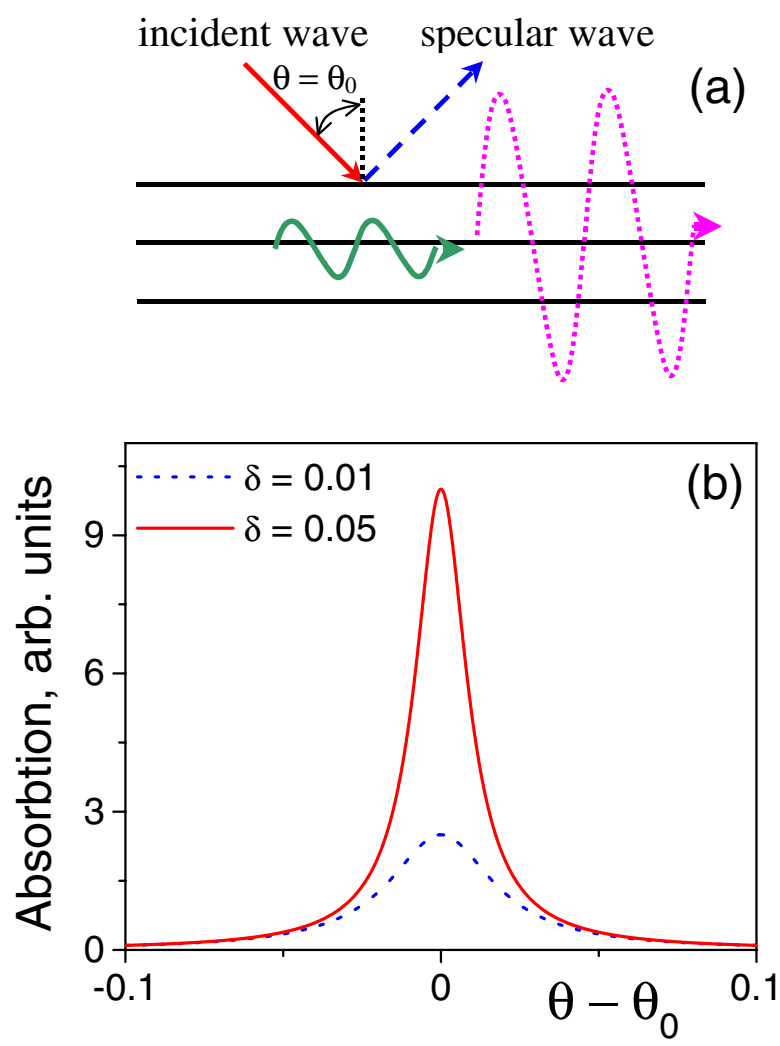

FIG. 2 (color online). (a) Schematic diagram showing the mechanism of excitation of the surface waves along the superconductor-vacuum interface. To zero-order approximation, with respect to the amplitude $\mu$ of the spatial modulations in a superconducting sample, an incident wave (shown as a solid red arrow) reflects as a specular wave (the straight dashed blue arrow), producing a damped wave (solid green wave) inside superconductors. To first order approximation, the very intense surface wave (dotted magenta wave) can be excited at a certain resonant angle between the incident wave and sample surface. (b) Absorption obtained using Eq. (18) for different effective dampings $\delta=\beta \omega_{r} \alpha \Omega^{4}\left(Q_{+}^{2}-\Omega^{2}\right)^{1 / 2}\left[2 \omega_{J}\left(1-\Omega^{2}\right)^{3} Q_{+} \cos \theta_{0}\right]^{-1}$.

To first order approximation, the solution of Eq. (4) with $\omega_{J}(x)$ inside the superconductor consists of both the forced component,

$$
H_{\text {forced }} \exp \left\{i q_{1} x-i \omega t-k_{-}\left(\frac{\omega \sin \theta}{c}, \omega\right) D l\right\},
$$

which attenuates with the same decrement as $H_{0}$, and free oscillations

$$
H_{\text {surf }} \exp \left\{i q_{1} x-i \omega t-k_{+}\left(q_{1}, \omega\right) D l\right\} .
$$

The amplitude $H_{\text {forced }}$ is defined by Eq. (4) itself, whereas the amplitudes, $H_{\text {surf }}$ (dotted magenta wave in Fig. 2) and corresponding vacuum mode

$$
H_{\text {surf }}^{\mathrm{vac}} \exp \left\{i q_{1} x-i \omega t-\left(q_{1}^{2}-\frac{\omega^{2}}{c^{2}}\right)^{1 / 2} z\right\}
$$

are determined by the boundary conditions for harmonics $q=q_{1}$ of $H$ and $E_{x}$ at $z=0$. Solving the corresponding 
set of nonhomogeneous linear equations for $H_{\text {surf }}$ and $H_{\text {surf }}^{\mathrm{vac}}$ we obtain the amplitude of the resonance wave:

$$
H_{\text {surf }}^{\mathrm{vac}}=H_{\mathrm{in}}^{\mathrm{vac}} \frac{2 \mu q_{0}^{2}}{\left(1-\Omega^{2}\right)\left(q_{1}^{2}-q_{0}^{2}\right)} \frac{1}{\mathcal{R}}
$$

with the denominator

$$
\mathcal{R}=X+i Y=\frac{2 Q_{+}(\Omega) \cos \theta_{0}\left(\theta-\theta_{0}\right)}{\beta \Omega \sqrt{Q_{+}^{2}(\Omega)-\Omega^{2}}}+\frac{i \omega_{r} \alpha \Omega^{3}}{\omega_{J}\left(1-\Omega^{2}\right)^{3}} .
$$

The resonance is characterized by the Lorentz form $1 / \mathcal{R}=1 /[X(\theta)+i Y]$. The excitation of the surface wave results in the resonant peak in the electromagnetic absorption,

$$
\operatorname{absorption}(\theta) \propto \sigma_{\perp} E_{z}^{2}+\sigma_{\|} E_{x}^{2} \propto \frac{1}{X^{2}(\theta)+Y^{2}},
$$

shown in Fig. 2. The resonance in the absorption can be observed by measuring the dependence of the surface impedance on the angle $\theta$. Alternatively, the peak in absorption produces a temperature increase, resulting in a sharp increase of the dc resistance or even the transition of the sample to the normal state at $\theta=\theta_{0}$.

Naturally, the absorption peak is accompanied by the resonant decrease of the amplitude $H_{\mathrm{sp}}^{\mathrm{vac}}$ of the specularly reflected wave. Even though this effect is of second order with respect to $\mu$, it can result in a significant (up to complete) suppression of $H_{\mathrm{sp}}^{\mathrm{vac}}$ at $\theta=\theta_{0}$ due to the resonance denominator $\mathcal{R}(\theta)$. In the optical range, this phenomenon is known as Wood's anomalies [13] of reflectivity and has been used for several diffraction devices.

$\mathrm{THz}$ detectors. - These effects could be potentially useful for the design of $\mathrm{THz}$ detectors, an important current goal of many labs worldwide. The simplest design could be a spatially modulated Bi2212 sample fixed on a precisely rotated holder and attached by contacts to measure its resistance. Spatial modulations in the sample could be fabricated by either using ion irradiation of the sample covered by periodically modulated mask [15], or even mechanically [16]. When rotating the sample, the incident $\mathrm{THz}$ radiation can produce a surface wave at certain angles. This results in a strong enhancement of absorption associated with increasing of temperature in the sample and, thus, its resistance. The relative positions of the resonance peaks (the set of angles) allows us to calculate the angle and the frequency of the incident $\mathrm{THz}$ radiation, while the relative heights of the resistance peaks can be used to estimate the intensity of the incident radiation.

Conclusions. - We have derived the surface Josephson plasma waves in layered superconductors and obtained their dispersion relation. The absorption of the incident electromagnetic wave can strongly increase at certain incident angles due to the resonant generation of the pre- dicted surface waves. This is the first prediction of propagating surface waves in any superconductor. We propose a way to experimentally observe these surface waves.

We acknowledge partial support from the NSA and ARDA under AFOSR Contract No. F49620-02-1-0334, and by the NSF Grant No. EIA-0130383.

[1] G. Blatter et al., Rev. Mod. Phys. 66, 1125 (1994); E. H. Brandt, Rep. Prog. Phys. 58, 1465 (1995).

[2] M. Tachiki, T. Koyama, and S. Takahashi, Phys. Rev. B 50, 7065 (1994); L.N. Bulaevskii, M.P. Maley, and M. Tachiki, Phys. Rev. Lett. 74, 801 (1995); M. Machida, T. Koyama, and M. Tachiki, Physica C (Amsterdam) 341, 1385 (2000); M. Tachiki and M. Machida, Physica C (Amsterdam) 341, 1493 (2000).

[3] Y. Matsuda et al., Phys. Rev. Lett. 75, 4512 (1995); Y. Matsuda et al., Phys. Rev. B 55, R8685 (1997); K. Lee et al., Phys. Rev. B 61, 3616 (2000); G. Hechtfischer et al., Phys. Rev. Lett. 79, 1365 (1997); K. Kadowaki, T. Wada, and I. Kakeya, Physica C (Amsterdam) 362, 71 (2001); K. Kadowaki et al., Phys. Rev. B 56, 5617 (1997).

[4] M. Tachiki et al., Phys. Rev. B 71, 134515 (2005); M. Machida et al., Physica C (Amsterdam) 362, 16 (2001); S. Savel'ev et al., Phys. Rev. B 72, 144515 (2005).

[5] See, e.g., the special issues: A. G. Davies, E. M. Linfield, and M. Pepper, Phil. Trans. R. Soc. A 362, 197 (2004); Special issue on Biomedical Imaging and Sensing Applications of THz Technology, edited by M. Chamberlain and M. Smith [Phys. Med. Biol. 47 (2002)].

[6] S.E. Shafranjuk, M. Tachiki, and T. Yamashita, Phys. Rev. B 55, 8425 (1997).

[7] Ch. Helm et al., Phys. Rev. Lett. 89, 057003 (2002).

[8] L. N. Bulaevskii et al., Europhys. Lett. 58, 415 (2002); Ch. Helm and L. N. Bulaevskii, Phys. Rev. B 66, 094514 (2002).

[9] S. Savel'ev, A. Rakhmanov, and F. Nori, Phys. Rev. Lett. 94, 157004 (2005).

[10] A. Gurevich and M. Tachiki, Phys. Rev. Lett. 83, 183 (1999); M. Machida, T. Koyama, and M. Tachiki, Phys. Rev. Lett. 83, 4618 (1999).

[11] P. M. Platzman and P. A. Wolff, Waves and Interactions in Solid State Plasmas (Academic, London, 1973).

[12] V.M. Agranovich and D. L. Mills, Surface Polaritons (Nauka, Moscow, 1985).

[13] H. Raether, Surface Plasmons (Springer, New York, 1988); R. Petit, Electromagnetic Theory of Gratings (Springer, Berlin, 1980); A. V. Kats and I. S. Spevak, Phys. Rev. B 65, 195406 (2002).

[14] W. L. Barnes, A. Dereux, and T.W. Ebbesen, Nature (London) 424, 824 (2003); A. V. Kats and A. Yu. Nikitin, Phys. Rev. B 70, 235412 (2004).

[15] W. K. Kwok et al., Physica C (Amsterdam) 382, 137 (2002).

[16] T. Tamegai et al., Physica C (Amsterdam) 412-414, 391 (2004). 